\title{
Internet-based guided self-help comprehensive behavioral intervention for tics (ICBIT) for youth with tic disorders: a feasibility and effectiveness study with 6 month-follow-up
}

\author{
Lilach Rachamim $^{1,2} \cdot$ Sharon Zimmerman-Brenner ${ }^{1,3} \cdot$ Osnat Rachamim $^{4} \cdot$ Hila Mualem $^{1,2} \cdot$ Netanel Zingboim $^{1} \cdot$ \\ Michael Rotstein ${ }^{4}$
}

Received: 27 August 2020 / Accepted: 11 November 2020 / Published online: 24 November 2020

(c) Springer-Verlag GmbH Germany, part of Springer Nature 2020

\begin{abstract}
Practice guidelines endorse comprehensive behavioral intervention for tics (CBIT) as first-line treatment for tic disorders (TD) in youth. Nevertheless, CBIT is rarely available due to various barriers. This study evaluated the feasibility and potential effectiveness of an Internet-based, self-help CBIT program (ICBIT) guided by parents with minimal therapist support delivered via telepsychotherapy. Forty-one youths, aged $7-18$ years, were randomly assigned to receive either ICBIT $(n=25)$ or a wait-list (WL) condition ( $n=16)$ in a crossover design. ICBIT was feasible to implement and at post-treatment, $64 \%$ of the participants have improved significantly. Results demonstrated clinically meaningful reductions in tic severity and improved youth global impairment and functioning. Gains were maintained over a 6-month follow-up period. The effect size for the primary outcome measure (Yale Global Tic Severity Scale) ranged between large effect size (Cohen"s $d=0.91$ ) at post-intervention to very large effect size (Cohen's $d=2.25$ ) 6 months after the end of the acute intervention. These were comparable to face-to-face delivery treatment trials for TD. Participants rated the intervention as highly acceptable and satisfactory. Youth receiving ICBIT experienced improvement in self-esteem and comorbidity. Finally, during the COVID19 pandemic, the ICBIT program enabled the delivery of the intervention consecutively without interruption. The results observed provide preliminary evidence of the feasibility and effectiveness of this innovative modality to assist youth with TD and remove various barriers to treatment, including those during a public crisis, such as the COVID-19 pandemic. Larger studies with an active control group are warranted.
\end{abstract}

Trial registration URL: http://clinicaltrials.gov, ClinicalTrials.gov Identifier: NCT04087616.

Keywords Child tic disorders - Comprehensive behavioral intervention for tics · Internet-based CBIT · Guided self-help · COVID-19 pandemic

Lilach Rachamim

rlilach@idc.ac.il

Sharon Zimmerman-Brenner

zbsharon@idc.ac.il

Osnat Rachamim osirach27@gmail.com

Hila Mualem hilm140@gmail.com

Netanel Zingboim zingboim.net@gmail.com

Michael Rotstein michaelr@tlvmc.gov.il
1 School of Psychology, Interdisciplinary Center Herzliya, Herzliya, Israel

2 Donald J. Cohen \& Irving B. Harris Resilience Center, Association for Children at Risk, Tel-Aviv, Israel

3 Tourette Syndrome Association in Israel (TSAI), Ra'anana, Israel

4 Pediatric Movement Disorders Clinic, Pediatric Neurology Unit, Dana-Dwek Children's Hospital - Tel Aviv Sourasky Medical Center, Tel Aviv, Israel 


\section{Introduction}

Primary tic disorders (TD) including Tourette syndrome (TS) and chronic tic disorder (CTD) are characterized by sudden, rapid, recurrent, non-rhythmic motor movements or vocalizations that are typically not context-related. Tics characteristically wax and wane in frequency and severity and are commonly preceded by an interoceptive sensation termed premonitory urge [1]. TS is a neurodevelopmental disorder defined by the presence of multiple motor and phonic tics that begins in childhood and persists for more than 1 year. Diagnosis of CTD is applied to children who exhibit motor or phonic tics, but not both, for at least a year [2]. The population prevalence estimate of CTD in youth is $1.5-3 \%$ and TS occurring in $0.3-0.9 \%$ of youth [3, 4]. Commonly associated comorbidities include attention-deficit hyperactivity disorder (ADHD; 29\%), obsessive-compulsive disorder (OCD; 19\%) and anxiety disorders including social phobia disorder (SPD; 21\%), and general anxiety disorder (GAD; 20\%) [5]. Studies suggest that TD may have negative ramifications on various spheres of life that may continue to adulthood [6-8]. Therefore, early and effective intervention of tic disorders is crucial.

Comprehensive behavioral intervention for tics (CBIT) [9], has gathered robust support from randomized controlled trials (RCTs) for its efficacy in improving tics [10]. Out of 126 children and adolescents (9-17 years old) receiving CBIT, 53\% demonstrated a clinically significant tic improvement compared with supportive therapy and education [11], and significant improvement in comorbid symptoms 6 months after treatment [12]. The efficacy of CBIT for young children (5-8 years old) was recently supported [13]. Despite the efficacy of CBIT, the vast majority of children with TD do not receive evidence-based interventions for tics [14] due to barriers, such as lack of trained therapists and personal and domestic considerations concerning time, distance and costs [15]. Remote administrations, such as tele-psychotherapy and internetbased self-help formats, have the potential to address some barriers in the dissemination of evidence-based interventions in the routine care of youth with TD. Such interventions have become more prominent during the COVID19 global pandemic of 2020, which has posed additional barriers to implementing psychotherapy and has enforced therapists to convert in-person therapy to online health service modalities. However, to date, only few pilot studies evaluated the feasibility and effectiveness of remote administrations of evidence-based treatments for youths with TD.

One pilot RCT conducted by Himle et al. [16] showed that CBIT delivered over videoconference (VC) was as effective as traditional face-to-face delivery. Another randomized WL-controlled pilot study, showed that CBIT delivered over VC was superior to a WL control group [17]. Only one pilot RCT showed that internet-based selfhelp programs were feasible, acceptable and effective in reducing tics in youth [18]. Twenty-three children were allocated to either internet-delivered habit reversal training (HRT) program (BIP TIC HRT) or internet-delivered exposure and response prevention (ERP) program (BIP TIC ERP), both were therapist-guided and parent-guided. Results indicated that while both interventions were effective in reducing tic-impairment, parent-rated tic severity and improved quality of life, only BIP TIC ERP resulted in a significant reduction on the Yale Global Tic Severity Scale (YGTSS) total tic severity score with gains maintained 12 months after intervention completion. While the study of Andrén et al. [18] adds to the growing evidence supporting the feasibility and potential efficacy of internet-delivered programs, further studies are needed to establish feasibility and effectiveness of internet-based self-help programs for pediatric TD and comorbid disorders. Whilst CBIT delivered via face-to-face and telehealth modalities has been previously established as an effective treatment for pediatric TD, internet-delivered CBIT (ICBIT) self-help program has not been yet evaluated in youth. To address this gap, we developed the first ICBIT Hebrew-language program guided by parents, and evaluated the feasibility, acceptability, durability and primary effectiveness of the program.

The aims of the present study were to: (1) evaluate the feasibility, acceptability and potential effectiveness of a novel parent-guided ICBIT program for improving tics in youth; (2) assess ICBIT effects on improving child's global impairment and functioning; (3) evaluate the effects of ICBIT on secondary outcomes including self-esteem, anxiety, attention deficit and impulsive symptoms, and obsessive-compulsive symptoms.

\section{Methods}

\section{Participants}

Participants were children and adolescents recruited through the pediatric movement disorders (PMD) clinic at DanaDwek Children's Hospital (DDCH), Sourasky medical center and the Tourette syndrome association in Israel. Inclusion criteria comprised a primary diagnosis of TS or CTD using the DSM-5 criteria [2]; Yale Global Tic Severity Scale; YGTSS [19] of mild to moderate (total tic severity score $>14$ ) in order for tic severity to be high enough to offer room for measurable change [11]; age between 7 and 18 years; at least one available parent to actively participate 
and support the child on regular basis throughout the program; agree to refrain from receiving other psychological treatment for TD; fluent Hebrew speakers; have access to a computer with Internet and web camera; children receiving psychotropic medications were eligible if the dose was stable for 6 weeks before and with no planned changes during participation in the study; children with comorbid symptoms of ADHD, anxiety, depression and OCD were allowed unless they required immediate intervention for such comorbid conditions. Exclusion criteria comprised a current diagnosis of substance abuse/dependence; a current diagnosis of major depression; suicidal risk; self-injurious tics; lifetime diagnosis of pervasive developmental disorder (PDD), mania and psychosis; intellectual developmental disorder (IDD) and previous sessions of CBIT.

\section{Procedure}

Broad inclusion and exclusion criteria were assessed via a phone screening interview conducted with the child's parent. The study was reviewed and approved by the Tel Aviv Sourasky Medical Center research ethics committee. The trial is registered on the National Institute for Health Research Portfolio Database (ClinicalTrials.gov Identifier: NCT04087616). All study procedures were conducted in accord with all necessary national and institutional regulations. The period of recruitment was 09/12/2018 to 09/12/2019. All parents and children signed an online informed consent form with a trained study staff member who provided information explaining the procedures and terms of the study. Participants were evaluated face-to-face at the PMD clinic at DDCH by a trained pediatric neurologist. The diagnosis of TS, CTD, PDD and IDD was obtained and clinically verified by DSM- 5 criteria [2]. After informed consent, eligible participants were invited to complete online questionnaires designed to assess tics and comorbid symptoms. Further information was obtained via video conference (VC) diagnostic interview by two $\mathrm{PhD}$ level clinicians who were blind to the experimental condition. Principal diagnosis of TS, CTD and comorbidity was confirmed by the DSM-5 criteria [2] as well as the YGTSS [19] and the Anxiety Disorders Interview Schedule; ADIS-C/P [20]. The combined reports of children and parents were used to establish diagnoses. Following assessment, families who met inclusion criteria were randomly allocated by a research assistant who was not otherwise involved in the study to one of two conditions: ICBIT or WL, using a 2:1 allocation ratio.

\section{Assessment}

Assessments were at baseline, post-ICBIT (9-weekly modules) or post-WL of equal duration. Directly after assessment, youths in the WL arm received ICBIT and were assessed at post-ICBIT, 3- and 6-months post-ICBIT completion. Figure 1 summarizes the flow of participants through the study. Out of the 92 children assessed for eligibility, 40 were excluded after a telephone screening interview with the parent and 11 were excluded after the diagnostic interview. Families excluded from the study were provided with appropriate referral information.

\section{Measures}

\section{Primary outcomes}

Yale Global Tic Severity Scale; YGTSS [19], is a semistructured clinical interview carried out with the parent and the child. The interview is composed of questions regarding motor and phonic tic severity over the previous week, followed by ratings, number, frequency, intensity and complexity of tics and degree of interference caused. Four composite scores are generated which are total motor tic severity (rated from 0 to 25), total phonic tic severity (rated from 0 to 25), total tic severity (composed of total motor and total phonic tic severity, rated from 0 to 50) and global YGTSS score (composed of total tic severity and impairment score, rated from 0 to 100). In the present study, inter-assessor reliability, determined from a random sample of $10 \%$ of participants, was 0.97 for the YGTSS ratings. YGTSS scores demonstrated strong correlations with Clinical global impression scale-severity $(r=0.82)$.

Clinical Global Impression-Improvement Scale (CGII), a clinician-rated scale was used to assess treatment response since baseline [21]. The CGI-I scale ranging from 1 (very much improved) to 7 (very much worse). In the present study, inter-assessor reliability, determined from a random sample of $10 \%$ of participants, was 0.99 for the CGI-I ratings. A score of 1 (very much improved) and 2 (much improved) was used to indicate a positive intervention response (at least "much improved").

Functional status was assessed with the clinician-rated Global Assessment Scale for Children; CGAS [22]. The CGAS provides a measure of global impairment and functioning over the previous month. Scores range from 1 (lowest functioning) to 100 (highest functioning). The CGAS possesses acceptable psychometric properties [23]. Scores on the CGAS were based on information gathered during the clinical interviews. In the present study, inter-assessor reliability, determined from a random sample of $10 \%$ of participants, was 0.96 for the CGAS ratings.

\section{Secondary outcomes}

Diagnostic status and symptom severity. Anxiety Disorders Interview Schedule; ADIS-C/P [20]. The ADIS child and 
Fig. 1 Subject flow through enrollment and follow-up

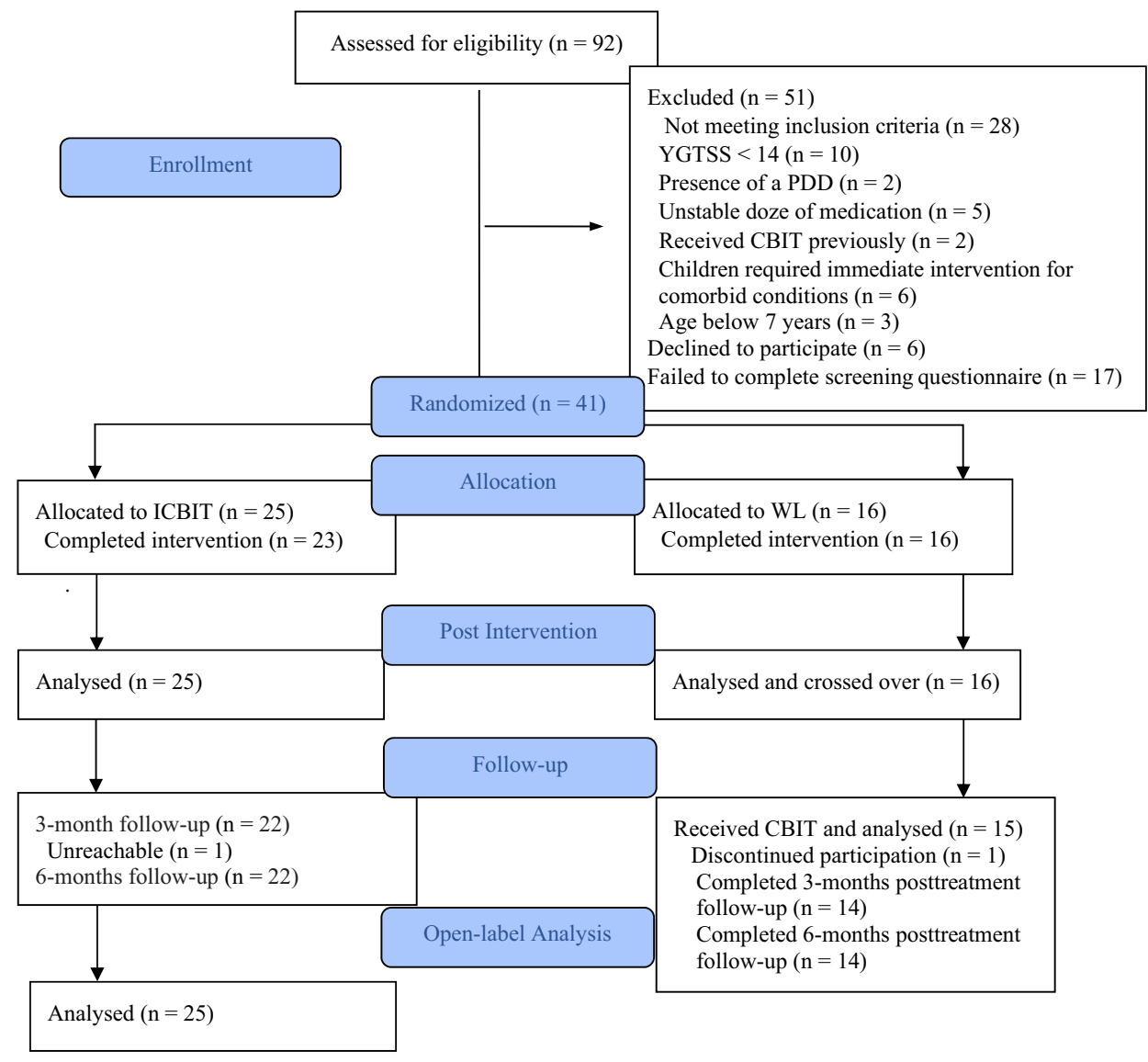

parent versions assesses anxiety, mood, and externalizing disorders in youth and screens for additional disorders.

Tic severity was measured through Parent Tic Questionnaire; PTQ [24]. Parents rate both the frequency and intensity of each tic on a 4-point scale. Each tic score is summed to yield overall scores for motor (RANGE $=0-112)$ and vocal (range $=0-112$ ) tics and a total score (range $=0-224)$. In the current study, the total PTQ score had good Cronbach's alpha (Cronbach's $\alpha=0.88$ ).

ADHD severity was measured by the Revised Connors' Parent Rating Scale; CPRS-R [25], a 48-item scale assessing symptom severity on a 4-point scale (range $=0-144)$. In the current study, the total score had good Cronbach's alpha (Cronbach's $\alpha=0.94$ ).

OCD comorbidity was measured through the Obsessive-Compulsive Inventory; OCI-CV [26], a 21-item scale assessing symptom severity on a 3-point scale (range $=0-42$ ). In the current study, the total OCI-CV score had good Cronbach's alpha (Cronbach's $\alpha=0.88$ ).

The Screen for Child Anxiety Related Emotional Disorders; SCARED [27], a 41-item measure assessing anxiety symptom severity on a 3 -point scale (range $=0-82$ ). The scale is completed separately by the child and his or her parent. In the current study, the total SCARED score had good Cronbach's alpha (Cronbach's $\alpha=0.88$ ).
The Liebowitz Social Anxiety Scale; LSAS [28], a 24-item measure assessing social anxiety and avoidance symptom on a 3-point scale (range $=0-144$ ). In the current study, the total LSAS score had good Cronbach's alpha (Cronbach's $\alpha=0.94$ ).

The Rosenberg Self Esteem Scale; RSES [29], a 10 -items rated on a 4-point scale. Higher scores indicate greater self-esteem (range $=10-40$ ). In the current study, the total RSES score had good Cronbach's alpha (Cronbach's $\alpha=0.81$ ).

The Children's Depression Inventory; CDI [30], a 27-item self-report scale that evaluates depression severity on a 3 -point scale (range $=0-81$ ). In the current study, the total CDI score had good Cronbach's alpha (Cronbach's $\alpha=0.81)$.

Satisfaction with the program and acceptability was assessed through a 3-item self-report questionnaire using a 0 (not at all), 1 (a little), 2 (fairly) 3 (very much) rating system for the program acceptability and perceived effectiveness: 1 . The program for managing tics was clear and I could implement it; 2 . The program for managing tics has improved tics; 3 . The program was helpful. Higher scores were indicative of greater satisfaction with treatment. 


\section{Intervention}

The ICBIT program was delivered through an internet program based on CBIT protocol [9] adapted to an interactive caregiver-guided self-help format. The main goals in the ICBIT program are to provide both child and caregiver with specific behavioral and cognitive skills for managing tics and facilitate the caregiver's support. The intervention consists of 9 consecutive conjoint child-caregiver modules, delivered over 9 weeks (see Table 1). Caregivers' only segments are included to facilitate their knowledge and skills to reinforce the child's coping and adherence to the program. Exercises for daily practice between modules provide the opportunity for practicing techniques that were practiced in each of the nine modules. ICBIT facilitates self-help therapy via ageappropriate texts and descriptive diagrams, animations, video clips of clinicians demonstrating techniques, such as competing response exercises and interactive tables. These interactive tables facilitate the tracking of progress and recording data about each tic (e.g., precipitating situations, behaviors and feelings experienced before tic, the number of tics et cetera). The intervention allows flexibility for the caregiver to present a module in one or more sittings depending on the child's attention span and to review previous units. Daily time spent on training is between 20 and $30 \mathrm{~min}$.

To enhance actual implementation of the program, improving the use of the skills and individualizing ICBIT strategies [31], throughout the intervention, two assistants provided weekly communication with the child and his or her parents. Children and parents were introduced to their assistant via a 15-20-min VC and received technical assistance in setting up the program. Weekly phone and VC calls were scheduled ahead to facilitate implementation of the program, providing assistance when necessary and gauging adverse outcomes. The assistants prompted program completion by providing feedback, praise and ensured accurate application of ICBIT skills. If the participants were not accessing the platform regularly, the assistant provided an additional telephone or VC call prompting youth and their parents to complete the module. The assistants checked module completion and adherence to the content of the module and sent reminders when the participant did not complete the tasks. In addition to supporting the child with his or her tasks, the assistants provided support to the parents (e.g., advise how to overcome the lack of his or her child's motivation, remind the parent to reward his or her child, organize and dedicate time for daily practice). The assistants were trainees (students in a Master's degree program in Clinical Psychology) with no previous experience in providing CBIT or ICBIT. Shortly before the intervention, they received brief training for the program and were familiarized with TD and related impairments. Three clinicians with considerable clinical experience in treating CBIT supervised the trainees regularly to provide guidance. All therapist time was logged. The average support time was $7.44 \mathrm{~min}(\mathrm{SD}=1.86)$ per family per week.

\section{Booster sessions}

Module 9 was repeated once a month as a monthly booster module for the next 6 months. During this time, a reminder was sent via email to remind participants to enter the platform to complete the booster session. In addition, the assistants reminded the participants to enter the booster module and scheduled a VC call to facilitate adherence and understanding of the booster module. When necessary, the assistants gave support to the child and the parent. Emphasis was placed on the importance of practicing the acquired skills on tics that may have been aggravated and implementing skills for other tics as well as new-onset tics.

Participants initially assigned to the WL, were instructed by the assistants they were to begin the ICBIT program once the initial WL period had ended. Participants in the WL arm received no intervention for 9 weeks and had no access to the ICBIT program during the WL period. Contact with the WL group was kept to a minimum (scheduling post-WL period assessment). Following post-WL assessment, all participants chose to receive ICBIT and received access to the ICBIT program.

\section{Adverse events}

Adverse events were monitored each week throughout the intervention. The assistants asked about recent tic worsening, behavioral changes, health complaints and visits for medical and psychological care, change in ongoing medications and need for concomitant medication and offered the opportunity for spontaneous report of any other problem. Confirmatory replies prompted further inquiry concerning the onset, severity and outcome of the adverse event and measures taken to address it.

\section{Statistical analysis}

All analyses were conducted using SPSS Version 25. Baseline characteristics were compared between groups with $t$ tests for continuous variables and $\chi^{2}$ tests for categorical variables. An intention-to-treat analysis was used. All participants assessed at baseline were included $(n=41)$, using last assessment carried forward for participants $(n=2)$ who were lost to post-intervention assessment, with all participants analyzed in their assigned group. A subsequent analysis included only participants who attended 4 or more sessions $(n=23)$ to provide a measure of effects when the program was adhered to treatment condition. A $2 \times 2$ mixeddesign repeated-measures analysis of variance (ANOVA) 
Table 1 Overview of the ICBIT Modules

Module 1

Module 2

Module 3

Module 4

Module 5-7

Module 8

Module 9
Psycho-education about tics and awareness training for tic occurrence ("Tic detectives"' team). Youth and their parents are taught to recognize their tics and increase awareness of precipitating and maintaining factors associated with tics. They are asked to monitor and record their tics daily (e.g., time of day, location and activity, the number of tics). Youth and their parents simply enter the data by clicking the interactive fields in the table. To keep children actively engaged in the program, parents are taught how to reword their child's efforts and record the points the child won.

Stress management skills (deep breathing and stress inoculation training and imagery).

Identifying factors that exacerbate tics (e.g., social settings, watching television) and developing functional strategies and day-to-day changes for reducing contact with contextual factors and improving coping skills in dealing with such factors. Coping skills and strategies are linked to a corresponding matrix that directs participants how and when to use them. For example, if tics are more prominent when watching television, less screen time is suggested as a strategy for reducing tics. Likewise, a child who starts tics more before an exam at school would be taught to manage his or her stress before and during the exam.

Education about a competing response and identifying premonitory urge for the first (out of 6) tic that the child identifies as his or hers most bothersome tics. Participants are introduced to perform a movement that is incompatible with the tic using antagonistic muscles (a "Tic blocker") that the child may apply when the urge for the tic is felt. A detailed list of 21 suggested competing responses for various motor and vocal tics and specifically created video clips, presenting a clinician specialized in CBIT for children, are used to illustrate choosing and practicing a range of competing responses. For example, a corresponding competing response for a shoulder shrug tic: "push shoulders downward to tighten shoulders and arms muscles against the shrugging movement". The child is taught to recognize the premonitory urge and begin pushing shoulders downward as a competing response.

Continue training for the second, third, fourth and the fifth tics (if a child has three tics or less, the guiding principles are to continue practicing the skills on the same tics).

Continue training for the sixth tic and generalization training. Participants practice in various daily situations including more challenging situations that may exacerbate tics (e.g., preparing for an exam, playing computer games).

Maintaining intervention gains and relapse prevention. Creating a "final project" (e.g., a simple folder detailing the methods and gains achieved and coping skills that may help to promote further improvement and relapse prevention); instructions and demonstrations for further practicing and weekly activities intendent to assist with maintenance and improvement (e.g., recording tics and setting new goals such as practicing the skills on additional tics, in various situations and during periods of tic exacerbation). test was used to examine main effects of group (ICBT or WL) as a between-subjects factor and time-point as a withinsubjects factor, as well as interaction effects. ANOVA tests were separately conducted for YGTSS total tic score (TTS) and impairment score (IS). Statistically significance for all analysis was set at the level of $p<0.05$ for a two-tailed test, and confidence intervals are given with $95 \%$ margin. A statistical power analysis, based on repeated-measures comparison $\left(\mathrm{G}^{*}\right.$ Power 3.1 .10$)$, between two groups indicated 
that a sample size of 41 participants would provide a power of 0.87 to detect an effect size of 0.25 at $\alpha=0.05$.

As all the families in the WL condition $(n=16)$ continued to the next assessment phase and crossed over to receive ICBIT, an open-trial analysis was conducted to increase power and examine effects of the ICBIT intervention for completers in the total sample (15 WL, 23 ICBIT) and maintenance of gains during the 6-month follow-up period. A series of repeated-measures ANOVA were conducted to investigate the maintenance of treatment effects from preICBIT (time 1) to post-ICBIT (time 2), to 3 (time 3 ) and 6-month (time 4 ) follow-up assessments. $F$ tests with Bonferroni corrections were performed.

\section{Results}

\section{Sociodemographic and diagnostic information}

Table 2 presents the sociodemographic and diagnostic information for the participants in each group and baseline group differences. Participants were 41 children and adolescents (12 girls) aged 7.89-17.57 years $(M=11.26, \mathrm{SD}=1.94)$ with a principal diagnosis of TS or CTD. All children were born in Israel. The majority of children (92.70\%) were living in families with both biological parents, and on average, children came from middle-income families as assessed through parental education level $(M=16.21 \mathrm{SD}=2.57)$ and parental employment status. No significant group differences in baseline characteristics were found on any variables except for specific phobia (SP). All participants $(n=4)$ who were diagnosed with SP were in the ICBIT arm. Out of the 25 youths allocated to ICBIT, 23 youths completed the 9-weekly modules. No medication changes were reported between baseline and post-intervention.

\section{ICBIT versus WL}

\section{Primary outcomes, module completion and safety}

Intention-to-treat analyses were performed $(n=25)$. Table 3 presents mean scores, effect sizes, and confidence intervals (CIs) on the difference between ICBIT and the WL control condition for all study outcomes. Module completion was monitored via the platform. Twenty-three families (92\%) completed all intervention modules. Participants completed a mean of $8.80(\mathrm{SD}=1.00)$ out of the 9 modules. Reasons for stopping $(n=2)$ included a lack of motivation and self-discipline.

Improvement rate (as indicated by a score of 1 and 2 on the CGI-I) was significantly higher for the ICBIT group $(16 / 25,64.00 \%)$ vs WL group $(1 / 16,6.25 \%)\left[\chi_{(1)}^{2}=13.40\right.$, $p<0.001]$.
A significant interaction was found for the YGTSS TTS between time-point and group $[F(1,39)=9.96, p=0.003$, $\mathrm{PES}=0.20$, large effect]. At post-intervention (time 2), the YGTSS TTS was significantly reduced in the ICBIT arm only. ICBIT was associated with a mean YGTSS TTS reduction of 6.60 points $(p<0.001)$ compared with a mean YGTSS TTS reduction of 0.94 points $(p=0.51)$ in the WL arm. This 6.60 points difference was clinically meaningful, as suggested by an effect size of within-group Cohen's $d=0.91$, large effect. For the YGTSS IS, a significant interaction was found between time-point and group $[F(1,39)=8.46, p=0.006, \mathrm{PES}=0.18$, large effect $]$. The YGTSS IS was significantly reduced in the ICBIT arm only $(p<0.001)$. ICBIT was associated with a mean reduction of 16.40 points compared with a 3.12-point decrease in the WL arm. The $29.05 \%$ mean decrease in clinical rated YGTSS TTS and the $48.81 \%$ decrease in the YGTSS IS were significantly greater than the WL group and fall between the ranges of large treatment effects. The Motor Tic Severity Score (MTS) significantly decreased $[F(1,39)=26.61$, $p<0.001$, PES $=0.40$, very large effect) $]$ The Vocal Tic Severity Score (VTS) did not decrease significantly $[F(1$, 39) $=0.12, p=0.73$, PES $=0.00]$.

Compared to youth in the WL condition, only youth in the ICBIT group experienced a significant improvement on the CGAS [65.60-72.20 vs 70.56-70.69, respectively; $F(1$, $39)=10.59, p=0.002 ; \mathrm{PES}=0.21]$. ICBIT was associated with a mean increase of 6.60 points on the CGAS scores compared with a 0.13-point increase in the WL arm.

A second analysis included only participants who attended 4 or more sessions $(n=23)$ to provide a measure of effects when the program was adhered to. There were no significant differences between the first and secondary analyses on all main outcomes.

\section{Follow-up}

The follow-up analysis included the participants in the CBIT arm. Intention-to-treat analyses were performed $(n=25)$. Out of 25 participants allocated to ICBIT, 22 completed the 6 booster modules and the follow-up assessments at 3 months and 6 months after the completion of the acute intervention. Results showed significant reductions during the follow-up period $[F(3,19)=28.29, p<0.001$; PES $=0.81$, very large effect]. At 3 months, the YGTSS TTS was associated with a mean reduction of 11.68 points $(p<0.001)$, Cohen's $d=2.11$, huge effect. At 6 months, the YGTSS TTS was associated with a mean reduction of 11.73 points ( $p<0.001)$, Cohen's $d=2.25$, very large effect. For the YGTSS IS, results showed significant reductions $[F(3$, 19) $=22.67, p<0.001 ; \mathrm{PES}=0.78$, very large effect $]$. At 3 months, the YGTSS IS was associated with a mean reduction of 27.73 points $(p<0.001)$, Cohen's $d=2.27$, very 
Table 2 Baseline demographic and clinical characteristics by group

\begin{tabular}{|c|c|c|c|c|c|}
\hline Measure & All $(n=41)$ & CBIT $(n=25)$ & WL $(n=16)$ & Statistic & $P$ \\
\hline Age, mean (SD) & $11.26(1.94)$ & $11.42(2.19)$ & $11.01(1.49)$ & $t_{(39)}=0.66$ & 0.51 \\
\hline \multicolumn{6}{|l|}{ Gender, mean (SD) } \\
\hline Males & $29(70.73 \%)$ & $17(68 \%)$ & $12(75 \%)$ & \multirow[t]{2}{*}{$\chi^{2}=0.23$} & \multirow[t]{2}{*}{0.63} \\
\hline Females & $12(29.26 \%)$ & $8(32 \%)$ & $4(25 \%)$ & & \\
\hline Maternal age, mean (SD) & $41.24(4.02)$ & $42.04(3.92)$ & $40(3.98)$ & $t_{(39)}=1.60$ & 0.11 \\
\hline Maternal years of education, mean (SD) & $16.21(2.57)$ & $16.36(2.3)$ & $16(3.01)$ & $t_{(39)}=0.43$ & 0.66 \\
\hline \multicolumn{6}{|l|}{ Maternal employment status, $n(\%)$} \\
\hline Full-time job & $22(53.65 \%)$ & $14(56.00 \%)$ & $8(50.00 \%)$ & \multirow{3}{*}{$\chi_{(2)}^{2}=1.53$} & \multirow[t]{3}{*}{0.46} \\
\hline Part-time job & $14(34.14 \%)$ & $7(28.00 \%)$ & $7(43.75 \%)$ & & \\
\hline Unemployed & $5(12.19)$ & $4(16.00 \%)$ & $1(6.25 \%)$ & & \\
\hline Paternal age, mean (SD) & $43.06(3.88)$ & $43.41(3.58)$ & $42.53(4.37)$ & $t_{(36)}=0.67$ & 0.50 \\
\hline Paternal years of education, mean (SD) & $15.4(2.7)$ & $15.82(2.82)$ & $14.86(2.5)$ & $t_{(36)}=1.06$ & 0.29 \\
\hline \multicolumn{6}{|l|}{ Paternal employment status, $n(\%)$} \\
\hline Full-time job & $33(80.48 \%)$ & $20(80.00 \%)$ & $13(81.25 \%)$ & \multirow[t]{3}{*}{$\chi_{(2)}^{2}=0.14$} & \multirow[t]{3}{*}{0.93} \\
\hline Part-time job & $3(7.31 \%)$ & $2(8.00 \%)$ & $1(6.25 \%)$ & & \\
\hline Unemployed & $2(4.87 \%)$ & $1(4.00 \%)$ & $1(6.25 \%)$ & & \\
\hline \multicolumn{6}{|l|}{ Current medication use, $n(\%)$} \\
\hline No & $31(75.60 \%)$ & $21(84 \%)$ & $10(62.5 \%)$ & \multirow[t]{7}{*}{$\chi_{(1)}^{2}=1.52$} & \multirow[t]{7}{*}{0.21} \\
\hline$\alpha$-agonists & $4(9.75 \%)$ & $1(4 \%)$ & $3(18.75 \%)$ & & \\
\hline Antipsychotics & $1(2.43 \%)$ & $1(4 \%)$ & - & & \\
\hline SSRI (Anxiety) & $1(2.43 \%)$ & - & $1(6.25 \%)$ & & \\
\hline Benzodiazepine & $1(2.43 \%)$ & $1(4 \%)$ & & & \\
\hline Stimulants (ADHD) & $2(4.87 \%)$ & $1(4 \%)$ & $1(6.25 \%)$ & & \\
\hline Medicinal-herbs & $1(2.6 \%)$ & - & $1(6.25 \%)$ & & \\
\hline \multicolumn{6}{|l|}{ Past psychotherapy experience, $n(\%)$} \\
\hline No & $13(31.7 \%)$ & $8(32 \%)$ & $5(31.25 \%)$ & \multirow[t]{6}{*}{$\chi_{(5)}^{2}=2.44$} & \multirow[t]{6}{*}{0.78} \\
\hline Psychotherapy & $12(29.26 \%)$ & $7(28 \%)$ & $5(31.25 \%)$ & & \\
\hline Expressive Arts Therapy & $7(17.07 \%)$ & $4(16 \%)$ & $3(18.75 \%)$ & & \\
\hline Occupational therapy & $5(12.19 \%)$ & $4(16 \%)$ & $1(6.25 \%)$ & & \\
\hline Animal-assisted therapy & $1(2.43 \%)$ & - & $1(6.25 \%)$ & & \\
\hline Acupuncture & $3(7.31 \%)$ & $2(8 \%)$ & $1(6.25 \%)$ & & \\
\hline \multicolumn{6}{|l|}{ Tic disorder, $n(\%)$} \\
\hline CTD & $9(21.95 \%)$ & $6(24 \%)$ & $3(18.75 \%)$ & $t_{(7)}=1.33$ & 0.21 \\
\hline TS & $31(75.6 \%)$ & $19(76 \%)$ & $13(81.25 \%)$ & $t_{(30)}=0.87$ & 0.50 \\
\hline $\mathrm{SAD}, n(\%)$ & $6(14.63 \%)$ & $3(12 \%)$ & $3(18.75 \%)$ & $\chi_{(1)}^{2}=0.35$ & 0.55 \\
\hline $\mathrm{GAD}, n(\%)$ & $16(39.02 \%)$ & $11(44 \%)$ & $5(31.25 \%)$ & $\chi_{(1)}^{2}=066$ & 0.41 \\
\hline $\mathrm{SPD}, n(\%)$ & $9(21.95 \%)$ & $6(24 \%)$ & $3(18.75 \%)$ & $\chi_{(1)}^{2}=0.15$ & 0.69 \\
\hline $\mathrm{SP}, n(\%)$ & $4(9.80 \%)$ & $4(16 \%)$ & - & $\chi_{(1)}^{2}=2.83$ & 0.04 \\
\hline OCD, $n(\%)$ & $13(31.70 \%)$ & $6(26.08 \%)$ & $7(43.75 \%)$ & $\chi_{(1)}^{2}=2.41$ & 0.29 \\
\hline Dysthymia, $n(\%)$ & $3(7.31 \%)$ & $3(12 \%)$ & - & $\chi_{(1)}^{2}=2.07$ & 0.15 \\
\hline Enuresis, $n(\%)$ & $3(7.31 \%)$ & $2(8 \%)$ & $1(6.25 \%)$ & $\chi_{(1)}^{2}=0.44$ & 0.83 \\
\hline Encopresis, $n(\%)$ & $1(2.43 \%)$ & $1(4 \%)$ & - & $\chi_{(1)}^{2}=0.65$ & 0.41 \\
\hline $\mathrm{ADHD}, n(\%)$ & $18(43.90 \%)$ & $10(39.13 \%)$ & $8(46.66 \%)$ & $\chi_{(1)}^{2}=3.96$ & 0.52 \\
\hline $\mathrm{LD}, n(\%)$ & $7(17.07 \%)$ & $4(16 \%)$ & $3(18.75 \%)$ & $\chi_{(1)}^{2}=0.052$ & 0.81 \\
\hline SMD, $n(\%)$ & $6(14.63 \%)$ & $4(16 \%)$ & $2(12.5 \%)$ & $\chi_{(1)}^{2}=0.09$ & 0.75 \\
\hline
\end{tabular}

$S A D$ separation anxiety disorder, $S P D$ social phobia disorder, $G A D$ general anxiety disorder; $S P$ specific phobia, $O C D$ obsessive-compulsive disorder and subclinical $\mathrm{OCD}, A D H D$ attention-deficit hyperactivity disorder, $L D$ learning disabilities, $S M D$ sensory modulation disorder 


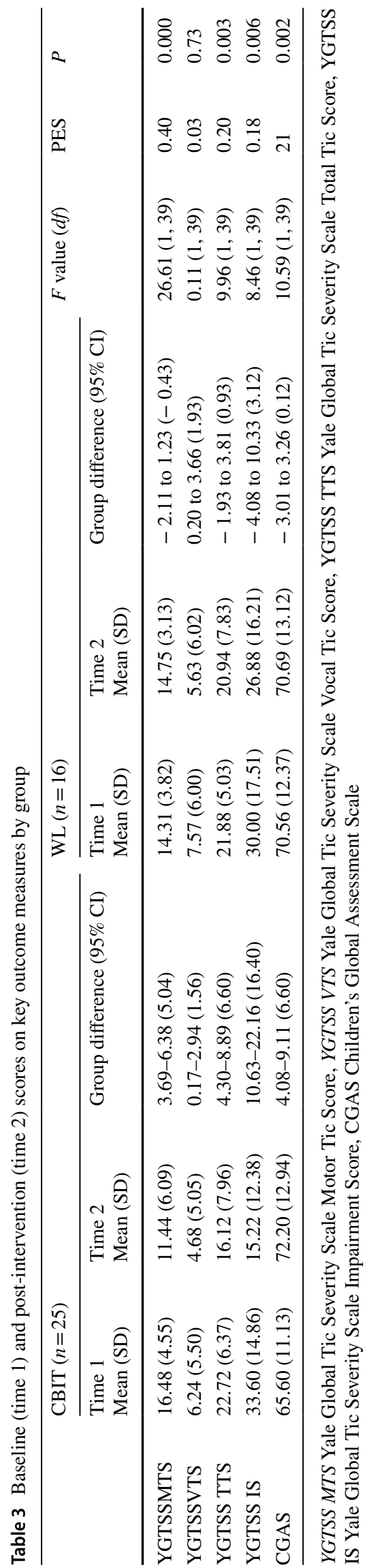

large effect. At 6 months, the YGTSS TTS was associated with a mean reduction of 28.64 points $(p<0.001)$, Cohen's $d=2.42$, very large effect. The YGTSS VTS yielded significant reductions during the follow-up period $[F(3,19)=3.74$, $p=0.03 ; \mathrm{PES}=0.37$, large effect $]$, and the YGTSS MTS also yielded significant reductions $[F(3,19)=60.56, p<0.001$; $\mathrm{PES}=0.90$, very large effect].

The results on the CGI-I showed that at 3-and 6-month follow-up assessments, $21 / 22,95.45 \%$, were rated as treatment responders (as indicated by a CGI-Improvement rating of "very much improved" or "much improved").

\section{Adverse events}

One adverse event was reported, rated as mild (high fever, $n=1)$ during the trial and the follow-up period. However, the event was not considered study related and no tic worsening was reported.

\section{COVID-19 pandemic}

Between March 15, 2020 and June 2020, the delivery of the intervention and assessments continued despite the quarantine imposed by the COVID-19 pandemic. Youths and their parents continued to log into the platform and participated in the clinical assessments delivered via VC.

\section{Open-trial analyses}

Table 4 displays baseline and mean scores, effect sizes, and confidence intervals (CIs) for all study outcomes. Participants in the open-trial analysis completed all nine modules. Two families (5.26\%) did not complete the booster-modules phase and did not return our calls. The results on the CGI-I showed that at post-intervention, 31/38, 81.57\% were rated as treatment responders. 3-month follow-up assessment, $33 / 36,91.66 \%$, and 6-month follow-up 34/36, $94.44 \%$, were rated as treatment responders.

At the end of the intervention, caregivers and children were asked to fill in a questionnaire regarding treatment and skills receipt. The questions referred to caregiver and child comprehension and perceived effectiveness of the ICBIT program, and caregiver and child satisfaction with the therapeutic outcomes. No intervention-related adverse events were reported. Results show that both children $(n=37)$ and parents $(n=37)$ gave high acceptability and satisfactory ratings. Mean child acceptability ratings were $M=7.89, \mathrm{SD}=1.21$. Mean parent acceptability ratings were $M=7.94, \mathrm{SD}=1.10$ (scale range $0-9$ ).

Youths exhibited a significant decrease in tic severity $[F(3,33)=54.25 p<0.001$, PES $=0.83$, very large effect]. From time 1 to time 2, YGTSS TTS decreased from 20.58 to $12.47,39.40 \%$ decrease. At time 3, there was 
Table 4 Baseline (time 1), post-intervention (time 2) and 3 (time 3) and 6-month (time 4) scores on primary and secondary outcome measures for ICBIT completers

\begin{tabular}{|c|c|c|c|c|c|c|c|}
\hline Measure & $\begin{array}{l}\text { Time } 0 \\
\text { Mean (SD) }\end{array}$ & $\begin{array}{l}\text { Time } 2 \\
\text { Mean (SD) }\end{array}$ & $\begin{array}{l}\text { Time } 3 \\
\text { Mean (SD) }\end{array}$ & $\begin{array}{l}\text { Time } 6 \\
\text { Mean (SD) }\end{array}$ & $F$ value $(d f)$ & PES & $P$ \\
\hline YGTSSMTS & $15.25(3.00)$ & $9.42(3.87)$ & $7.19(4.02)$ & $6.78(3.89)$ & $88.74(3,33)$ & 0.89 & 0.001 \\
\hline YGTSSVTS & $5.08(4.80)$ & $3.06(3.74)$ & $2.25(3.52)$ & $1.94(2.66)$ & $5.25(3,33)$ & 0.32 & 0.004 \\
\hline YGTSS TTS & $20.58(5.47)$ & $12.47(5.56)$ & $9.36(5.84)$ & $8.72(5.12)$ & $54.25(3,33)$ & 0.83 & 0.000 \\
\hline YGTSS IS & $30.00(14.41)$ & $13.06(11.11)$ & $5.83(9.41)$ & $4.72(3.89)$ & $33.81(3,33)$ & 0.75 & 0.000 \\
\hline CGAS & $68.47(12.40)$ & $75.44(13.05)$ & $78.83(12.74)$ & $80.64(12.87)$ & $17.34(3,33)$ & 0.61 & 0.000 \\
\hline PTQ & $31.17(15.93)$ & $18.39(14.54)$ & $14.61(11.30)$ & $11.97(14.84)$ & $14.93(3,33)$ & 0.57 & $0 . .000$ \\
\hline CRS-R & $30.22(18.07)$ & $23.33(17.01)$ & $22.14(14.63)$ & $19.68(15.41)$ & $10.34(3,33)$ & 0.48 & 0.000 \\
\hline SCARED: Parent & $16.56(9.79)$ & $12.61(9.34)$ & $10.56(8.55)$ & $6.06(7.32)$ & $12.17(3,33)$ & 0.52 & 0.000 \\
\hline SCARED: Child & $21.92(12.19)$ & $15.72(11.36)$ & $13.22(11.16)$ & $7.86(9.65)$ & $16.94(3,33)$ & 0.60 & 0.000 \\
\hline RSE: child & $34.14(4.22)$ & $35.39(4.48)$ & $35.58(3.43)$ & $23.08(16.07)$ & $14.73(3,33)$ & 0.57 & 0.000 \\
\hline CDI: child & $8.78(7.06)$ & $5.22(5.37)$ & $4.78(5.40)$ & $9.94(9.38)$ & $15.60(3,33)$ & 0.58 & 0.000 \\
\hline LSAS: child & 34.50 (21.69) & $23.72(23.66)$ & $21.05(22.00)$ & $17.72(22.72)$ & $6.83(3,33)$ & 0.38 & 0.001 \\
\hline OCI-CV: child & $11.31(6.33)$ & $6.81(6.04)$ & $6.97(6.62)$ & $7.00(7.13)$ & $6.16(3,33)$ & 0.36 & 0.002 \\
\hline
\end{tabular}

YGTSS MTS Yale Global Tic Severity Scale Motor Tic Score, YGTSS VTS Yale Global Tic Severity Scale Vocal Tic Score, YGTSS TTS Yale Global Tic Severity Scale Total Tic Score, YGTSS IS Yale Global Tic Severity Scale Impairment Score, CGAS Children's Global Assessment Scale, $P T Q$ Parent Tic Questionnaire, $C R S-R$ Conner's Rating Scales-Revised, SCARED Screen for Child Anxiety Related Emotional Disorders, $R S E$ Rosenberg Self Esteem Scale, CDI Children's Depression Inventory, LSAS Liebowitz Social Anxiety Scale, OCI-CV Obsessive-Compulsive Inventory-Childe Version

an additional reduction of $3.11,24.04 \%$ decrease, and at time 4 , there was an additional reduction of $0.64,6.15 \%$ decrease. For the YGTSS IS, there was a significant reduction $[F(3,33)=33.81, p<0.001, \mathrm{PES}=0.75$, very large effect]. From time 1 to time 2, scores decreased from 30.00 to $13.06,56.47 \%$ decrease, and from time 2 to time 3 , from 13.06 to $5.83,55.36 \%$ decrease, and from time 3 to time 4 , from 5.83 to $4.72,19.04 \%$ decrease.

Youth exhibited a significant decrease in VTS $[F(3$, $33)=5.25 p=0.004$, PES $=0.32$, large effect $]$ as well as in $\operatorname{MTS}[F(3,33)=88.74 p<0.001$, PES $=0.89$, very large effect].

Two children had medication changes during the followup period. Long-acting amphetamine was prescribed to relieve ADHD symptoms 2 months before time- 4 assessment and one child stopped taking antipsychotics 2 months before time- 4 assessment. The YGTSS TTS score of the first child continued to improve with an 18-point decrease from time 3 to time 4, and a 2-point increase in YGTSS TTS score of the second child. The analyses were repeated excluding these children with no significant changes seen in the results.

Youth exhibited a significant increase on the CGAS scale $[F(3,33)=17.34 p<0.001, \operatorname{PES}=0.61$, very large effect]. From time 1 to time 2, CGAS scores increased from 68.47 to $75.44,9.25 \%$ improvement, from time 2 to time 3 , from 75.44 to $78.83,4.31 \%$ improvement, and from time 3 to time 4 , from 78.83 to $80.64,2.25 \%$.

\section{Secondary outcomes}

The PTQ total tic scores from the parent-rated scale showed that youth exhibited significant tic severity decrease $[F(3,33)=14.93 p<0.001$, PES $=0.57$, very large effect]. From time 1 to time 2, there was a $41.01 \%$ decrease, a $20.56 \%$ decrease from time 2 to time 3 , and an $18.07 \%$ decrease from time 3 to time 4 .

Significant reductions were reported on the CPRS-R rating scale $[F(3,33)=10.34 p<0.001, \mathrm{PES}=0.48$, large effect].

Youths reported a significant large mean decrease in SCARED scores $[F(3,33)=16.94 p<0.001$, PES $=0.60$, very large effect]. Parent-reported SCARED scores also showed significant reductions $[F(3,33)=12.17 p<0.001$, $\mathrm{PES}=0.52$, very large effect $]$.

Youths experienced significant reductions on the OCI$\mathrm{CV}[F(3,33)=6.16 p=0.002, \mathrm{PES}=0.36$, large effect $]$.

Youths reported a significant mean increase in the RSE scores $[F(3,33)=14.73 p<0.001$, PES $=0.57$, very large effect].

Significant reductions were reported on the child rating LSAS scale $[F(3,33)=6.83 p=0.001, \mathrm{PES}=0.38$, large effect].

Youths reported a significant large mean decrease in CDI scores $[F(3,33)=15.60 p<0.001, \operatorname{PES}=0.58$, very large effect]. See Table 4. 


\section{Discussion}

The present study presents original data on the feasibility and preliminary effects of an internet-based CBIT self-help program guided by parents, with brief remote support by a therapist for Israeli youth with TD. The outcome data of this study indicated that ICBIT was feasible as demonstrated by the high retention rate (92\%). The dropout rate of $8 \%$ in this study is very encouraging and is similar to the reported rates in studies of face-to-face [11] and VC delivery of CBIT [16]. Based on the study of Radomski et al. [31], several delivery features in self-help internet-based programs may have led to the high retention rate in the current study: (1) social role-an adjunct therapist support to facilitate the use and prompting as well as praising the participant's completion and progress; (2) Flexibility - the program allows children and their parents to engage in the intervention when it is convenient for them without missing school or work. The contribution of the social role supporting self-help intervention for youth with TD was recently reported and suggest that adjunct therapist support may prompt adherence to treatment and improve the efficacy of the intervention [32]. The results suggest that this avenue of administration is safe as no unusual events have been reported due to the use of this intervention modality. The program was associated with high levels of satisfaction among participants. Furthermore, the intervention was cost-effective since the average therapist support time was around 8 min per participant per week.

Following ICBIT, there was a significant reduction in motor tic severity and in impairment scores whereas participants in the WL group showed no improvement. Although the present study did not include a comparison to an active treatment condition, the findings are promising in showing large effects on motor tics as well as on global level of functioning that are comparable with CBIT administered in an in-person, clinic-based setting [11]. The reductions found in the current study are higher than the $25 \%$ considered clinically meaningful by Jeon et al. [33]. ICBIT yielded a mean YGTSS TTS reduction of 6.6 points $(29.15 \%)$ and a mean YGTSS IS reduction of 14.80 points $(44.05 \%)$ from baseline to post-intervention, which is comparable to the 7.6 points $(31 \%)$ and 12.8 points $(51 \%)$ reductions reported by Piacentini et al. [11]. The tic reduction as well as response rate observed in the present study is comparable to what was observed in previous trials incorporating face-to-face and VC delivery as well as self-help programs $[11,16,18]$.

The follow-up analyses showed further significant improvements in motor tic severity as well as significant improvement in vocal tic severity between baseline and 3-to-6 month follow-ups. It is plausible that the six booster modules in the current study were associated with outcome improvement as was previously found in the study of Heijerman-Holtgrefe et al. [34]. Furthermore, during the follow-up period, participants were instructed to implement their recent acquired skills on additional tics. Participants could go back and watch the video illustrations for practicing a range of competing responses in various situations and practice relaxation through accessible audio clips. It is conceivable that the availability of the program, as well as the booster sessions, allowed for generalization and enhanced further improvement during the follow-up period.

Comparable to motor tics, vocal tics did not significantly improve following the acute phase of the intervention (time 2). Nevertheless, vocal tics did significantly improve during the follow-up phase. In the current study, out of the 25 youths allocated to ICBIT, 17 had vocal tics. Only eight youths ranked vocal tics as the most distressing tic and chose to address them in the acute phase. During the follow-up phase, vocal tics improved significantly. This improvement may be attributed to further practicing and booster sessions during the follow-up period. It is conceivable that 8 youths constituted a small sample size to obtain a significant finding. However, the delayed impact of the intervention on vocal tics compared to motor tics may be attributed to different neurobiological substrates [35] and to vocal tics sensitivity to environmental effects [36].

The open-trial analysis yielded significant effects of ICBIT on improving self-esteem as was previously found in adults with TD [37] and on comorbid symptoms of anxiety found in youth with TD [12]. Additionally, although ICBIT is not designed to improve symptoms of OCD, anxiety and ADHD, the program yielded significant improvement in OCD, ADHD, social anxiety as well as depressive symptoms. These findings may suggest that several components of ICBIT, such as relaxation techniques, may regulate comorbid symptoms [12]. Nevertheless, ICBIT is not intended to replace evidence-based treatment practices for ADHD, $\mathrm{OCD}$, and anxiety disorders.

Taken together, our results suggest that internet self-help CBIT program for youth with TD, guided by caregivers with limited remote professional support, offers an acceptable and safe alternative that may facilitate wide accessibility to families who face barriers to accessing evidenced-based therapy for TD.

The outbreak of the COVID-19 global pandemic has confronted the healthcare systems with unprecedented challenges. Worldwide containment efforts embrace isolation, quarantine and social distancing. Nevertheless, the ICBIT program and videoconferencing allowed the delivery of the intervention consecutively. During the COVID-19 pandemic, we continued to support and monitor the participants' implementation of the ICBIT intervention as usual. Thus, ICBIT has the potential to enhance accessibility of effective interventions for youth with TD. 


\section{Strengths and limitations}

Several limitations should be noted. The study consisted of a small, yet compelling, sample size of Israeli youth. The present study did not include youth with minimal impairment (total severity scores $<14$ ) and youth with marked impairment scores (total severity scores $>40$ ). Children under the age of 7 years old were not included. In addition, the study did not include a non-CBIT active treatment control group. These may restrict generalizability of the results. It would be important to replicate this trial with a larger, and more heterogenic sample, and compare ICBIT with traditional face-toface delivery. While the average therapist time was logged, the parental support time was not logged in the current study. Additional studies on the contribution of parental support for the efficacy of self-help ICBIT are warranted. Further research is needed to advance our knowledge on moderators and predictors of response to ICBIT to help identify those individuals who may not benefit from internet-delivered CBIT. This area of exploration is in its infancy and future studies should aim to compare the efficacy and long-term maintenance of gains made between internet-based CBIT modalities in a larger cohort of patients.

Despite the previously mentioned study limitations, the present RCT was the first to evaluate the feasibility and efficacy of ICBIT. Additional well-controlled and empiricallybased treatment outcome studies are warranted.

Acknowledgements The authors would like to thank families who participated and the Tourette Syndrome Association in Israel (TSAI) who helped recruit participants.

Author contributions LR: research project: conception, planning, adaptation of CBIT to an internet format, organization, execution, design, statistical analysis, manuscript: co-writing of the first draft, review and critique, contributed to the final version of the manuscript. SZ-B: research project: adaptation of CBIT to an internet format, execution; statistical analysis, review and critique; manuscript: co-writing of the first draft, review and critique, contributed to the final version of the manuscript. OR: research project: conception, adaptation of CBIT to an internet format, organization; manuscript: co-writing of the first draft, review and critique, contributed to the final version of the manuscript. HM: research project: organization, data collection, statistical analysis, manuscript: contributed to the final version of the manuscript. NZ: research project: data collection, execution, statistical analysis. MR: research project: conception, organization, execution; manuscript: co review and critique, contributed to the final version of the manuscript.

\section{Compliance with ethical standards}

Conflict of interest None declared by all authors.

Ethics approval All procedures performed in studies involving human participants were in accordance with the ethical standards of the Institutional Committee at Tel Aviv Sourasky Medical Center and with the 1975 Helsinki Declaration and its later amendments or comparable ethical standards (IRB 0815-18-TLV).
Consent to participate Informed consent was obtained from all the patients included in the study prior to enrollment.

Consent for publication Each of the authors contributed to the work, and have seen and agreed with the contents of the manuscript. All authors have agreed to submit the manuscript.

\section{References}

1. Ganos C, Garrido A, Navalpotro-Gómez I et al (2015) Premonitory urge to tic in Tourette's is associated with interoceptive awareness. Mov Disord 30:1198-1202. https://doi.org/10.1002/ mds. 26228

2. American Psychiatric Association (2013) Diagnostic and statistical manual of mental disorders: DSM-5. American Psychiatric Association, Arlington

3. Kurlan R, McDermott MP, Deeley C et al (2001) Prevalence of tics in schoolchildren and association with placement in special education. Neurology 57:1383-1388. https://doi.org/10.1212/ WNL.57.8.1383

4. Scharf JM, Miller LL, Gauvin CA et al (2015) Population prevalence of Tourette syndrome: a systematic review and meta-analysis. Mov Disord 30:221-228. https://doi.org/10.1002/mds.26089

5. Specht MW, Woods DW, Piacentini J et al (2011) Clinical characteristics of children and adolescents with a primary tic disorder. J Dev Phys Disabil 23:5-31. https://doi.org/10.1007/s1088 2-010-9223-Z

6. Lewin AB, Murphy TK, Storch EA et al (2012) A phenomenological investigation of women with Tourette or other chronic tic disorders. Comp Psychiatry 53:525-534. https://doi.org/10.1016/j. comppsych.2011.07.004

7. Altman G, Staley JD, Wener P (2009) Children with Tourette disorder: a follow-up study in adulthood. J Nerv Ment Dis 197:305310. https://doi.org/10.1097/NMD.0b013e3181a206b1

8. Cavanna AE, David K, Bandera V et al (2013) Health-related quality of life in Gilles de la Tourette syndrome: a decade of research. Behav Neurol 27:83-93. https://doi.org/10.3233/BEN120296

9. Woods DW, Piacentini J, Chang S et al (2008) Managing Tourette syndrome: a behavioral intervention for children and adults therapist guide. Oxford University Press, Oxford

10. McGuire JF, Piacentini J, Brennan EA et al (2014) A meta-analysis of behavior therapy for Tourette syndrome. J Psychiatr Res 50:106-112. https://doi.org/10.1016/j.jpsychires.2013.12.009

11. Piacentini J, Woods DW, Scahill L et al (2010) Behavior therapy for children with Tourette disorder: a randomized controlled trial. JAMA Psychiatry 303:1929-1937. https://doi.org/10.1001/ jama.2010.607

12. Woods DW, Piacentini JC, Scahill L et al (2011) Behavior therapy for tics in children: acute and long-term effects on psychiatric and psychosocial functioning. J Child Neurol 26:858-865. https://doi. org/10.1177/0883073810397046

13. Bennet SM, Capriotti M, Bauer C et al (2020) Development and open trial of a psychosocial intervention for young children with chronic tics: the CBIT-JR study. Behav Ther 51:659-669. https:// doi.org/10.1016/j.beth.2019.10.004

14. Hollis C, Pennant M, Cuenca J et al (2016) Clinical effectiveness and patient perspectives of different treatment strategies for tics in children and adolescents with Tourette syndrome: a systematic review and qualitative analysis. Health Technol Assess 20:1-450. https://doi.org/10.3310/hta20040 
15. Woods DW, Conelea CA, Himle MB (2010) Behavior therapy for Tourette's disorder: utilization in a community sample and an emerging area of practice for psychologists. Prof Psychol Res Pract 41:518-525. https://doi.org/10.1037/a0021709

16. Himle MB, Freitag M, Walther M et al (2012) A randomized pilot trial comparing videoconference versus face-to-face delivery of behavior therapy for childhood tic disorders. Behav Res Ther 50:565-570. https://doi.org/10.1016/j.brat.2012.05.009

17. Ricketts EJ, Goetz AR, Capriotti MR et al (2016) A randomized waitlist-controlled pilot trial of voice over Internet protocoldelivered behavior therapy for youth with chronic tic disorders. J Telemed Telecare 22:153-162. https://doi.org/10.1177/13576 33X15593192

18. Andrén P, Aspvall K, Fernández de la Cruz L et al (2019) Therapist-guided and parent-guided internet-delivered behavior therapy for pediatric Tourette's disorder: a pilot randomized controlled trial with long term follow-up. BMJ Open 15:9. https://doi. org/10.1136/bmjopen-2018-024685 ((e024685))

19. Leckman JF, Riddle MA, Hardin MT et al (1989) The Yale Global Tic Severity Scale: initial testing of a clinician-rated scale of tic severity. J Am Acad Child Adolesc Psychiatry 28:566-573. https ://doi.org/10.1097/00004583-198907000-00015

20. Silverman WK, Nelles WB (1988) The anxiety disorders interview schedule for children. J Am Acad Child Adolesc Psychiatry 27:772-778. https://doi.org/10.1097/00004583-198811000-00019

21. Guy W (1976) Clinical global impressions, ECDEU Assessment Manual for Psycho-pharmacology. National Institute for Mental Health, Rockville, pp 218-222

22. Shaffer D, Gould MS, Brasic J et al (1983) A Children's global assessment scale (CGAS). Arch Gen Psychiatry 40:1228-1231. https://doi.org/10.1001/archpsyc.1983.01790100074010

23. Green B, Shirk S, Hanze D, Wanstrath J (1994) The children global assessment scale in clinical practice. J Am Acad Child Adolesc Psychiatry 33:1158-1164. https://doi.org/10.1097/00004 583-199410000-00011

24. Chang S, Himle MB, Tucker BTP et al (2009) Initial psychometric properties of a brief parent-report instrument for assessing tic severity in children with chronic tic disorders. Child Fam Behav Ther 31:181-191. https://doi.org/10.1080/07317100903099100

25. Goyette CH, Conners CK, Ulrich RF (1978) Normative data on revised Conners parent and teacher rating scales. J Abn Child Psychol 6:221-236. https://doi.org/10.1007/BF00919127

26. Foa EB, Coles M, Huppert JD et al (2010) Development and validation of a child version of the obsessive-compulsive inventory. Behav Ther 41:121-132. https://doi.org/10.1016/j. beth.2009.02.001
27. Birmaher B, Khetarpal S, Brent D et al (1997) The screen for child anxiety related emotional disorders (SCARED): scale construction and psychometric characteristics. J Am Acad Child Adolesc Psychiatry 36:545-553. https://doi.org/10.1097/00004583-19970 4000-00018

28. Liebowitz MR (1987) Social phobia. Mod Probl Pharmacopsychiatr 22:141-173. https://doi.org/10.1159/000414022

29. Rosenberg M (1965) Society and the adolescent self-image. Princeton University Press, Princeton

30. Kovacs M (1985) The Children's Depression, Inventory (CDI). Psychopharmacol Bull 21(4):995-998

31. Radomski AD, Wozney L, McGrath P et al (2019) Design and delivery features that may improve the use of internet-based cognitive behavioral therapy for children and adolescents with anxiety: a realist literature synthesis with a persuasive systems design perspective. J Med Internet Res 21:e11128. https://doi. org/10.2196/11128

32. Chamberlain LR, Hall CL, Andrén P et al (2020) Therapist-supported online interventions for children and young people with tic disorders: lessons learned from a randomized controlled trial and considerations for future practice. JMIR Ment Health 7:e19600. https://doi.org/10.2196/19600

33. Jeon S, Walkup JT, Woods DW et al (2013) Detecting a clinically meaningful change in tic severity in Tourette syndrome: a comparison of three methods. Contemp Clin Trials 36:414-420. https ://doi.org/10.1016/j.cct.2013.08.012

34. Heijerman-Holtgrefe AP, Verdellen CWJ, van de Griendt JMTM et al (2020) Tackle your tics: pilot findings of a brief, intensive group-based exposure therapy program for children with tic disorders. Eur Child Adolesc Psychiatry. https://doi.org/10.1007/ s00787-020-01532-5.Advanceonlinepublication.10.1007/s0078 7-020-01532-5

35. McCairn KW, Nagai Y, Hori Y et al (2016) A primary role for nucleus accumbens and related limbic network in vocal tics. Neuron 89:300-307. https://doi.org/10.1016/j.neuron.2015.12.025

36. Goldman KJ, DeLeon IG (2020) A review on functional analyses of tics. Behav Modif 44:296-315. https://doi.org/10.1177/01454 45518809046

37. Weingarden E, Scahill L, Hoeppner S et al (2018) Self-esteem in adults with Tourette syndrome and chronic tic disorders: The roles of tic severity, treatment, and comorbidity. Compr Psychiatry 84:95-100. https://doi.org/10.1016/j.comppsych.2018.04.008 